La revue La revue pour l'histoire du CNRS

$++++2007$

Objectif biotechs?

Biotechs : de la recherche académique au monde industriel

Le rôle du Club Ecrin Biotechnologies

Françoise Xavier

(2) OpenEdition

Journals

Édition électronique

URL : https://journals.openedition.org/histoire-cnrs/1976

DOI : 10.4000/histoire-cnrs. 1976

ISSN : 1955-2408

Éditeur

CNRS Éditions

Édition imprimée

Date de publication : 3 juillet 2007

ISBN : 978-2-271-06558-2

ISSN : 1298-9800

Référence électronique

Françoise Xavier, «Biotechs : de la recherche académique au monde industriel », La revue pour l'histoire du CNRS [En ligne], 17 | 2007, mis en ligne le 03 juillet 2009, consulté le 20 mai 2021. URL : http://

journals.openedition.org/histoire-cnrs/1976 ; DOI : https://doi.org/10.4000/histoire-cnrs.1976

Ce document a été généré automatiquement le 20 mai 2021.

Comité pour l'histoire du CNRS 


\title{
Biotechs : de la recherche académique au monde industriel
}

\author{
Le rôle du Club Ecrin Biotechnologies
}

\section{Françoise Xavier}

1 La revue pour l'histoire du CNRS. Pouvez-vous retracer brièvement votre parcours professionnel?

2 Françoise Xavier. J'ai fait toute ma carrière au CNRS. J'ai passé 25 ans à l'École normale supérieure-Ulm (Ens-Ulm), d'abord comme doctorante, puis comme responsable d'équipe. L'orientation générale de mes recherches a été déterminée lorsque je suis devenue, à l'École normale supérieure, l'élève du professeur Maxime Lamotte, à la fois généticien, zoologiste et écologiste.

3 J'ai ainsi pris conscience très tôt, dès les années 1960, de l'importance de la pluridisciplinarité, du travail d'équipe et entre équipes, de la nécessité de savoir maîtriser de nouvelles méthodologies dans de nouvelles disciplines et acquérir des financements publics et/ou privés pour le développement de projets spécifiques de recherche, enfin de privilégier la mobilité physique lorsqu'elle devient indispensable à l'émergence et à l'évolution dynamique de nouvelles recherches.

4 J'ai acquis très vite des responsabilités non seulement de formation d'étudiants à la recherche au sein du laboratoire, mais aussi d'enseignement. C'est ainsi que pendant vingt ans j'ai assuré un enseignement pratique et théorique à l'agrégation de sciences naturelles de l'Ens, qui a été accompagné ou suivi de charges de cours de maîtrise à l'université Paris VI et à l'université de Versailles Saint-Quentin-en-Yvelines.

5 L'évolution de mes recherches et des technologies en biologie moléculaire et cellulaire m'a amenée ensuite à diriger des équipes à l'Inra et de nouveau à l'Ens-Ulm, dans le cadre de la recréation d'une unité Inserm affiliée au CNRS. Depuis mars 2005, j'ai intégré le Laboratoire de mathématiques appliquées aux systèmes de l'École centrale Paris pour développer des projets de recherche entre mathématiciens, biologistes, biophysiciens, médecins et, pour certains, industriels au sein de mon équipe. 
6 La revue... Pourquoi avez-vous rejoint l'association Ecrin ? Vous étiez directrice de recherche, l'enjeu n'était pas fondamental pour votre carrière. Avez-vous été missionnée? Par la direction générale de la recherche?

7 F.X. J'ai fondé ma carrière scientifique sur la mobilité intellectuelle, qui entraîne la pluridisciplinarité, et l'ai associée à une mobilité physique, lorsque celle-ci devenait indispensable à l'émergence et à l'évolution dynamique de nouvelles recherches. Dès les années 1980, j'ai réalisé que la mise en œuvre de la diversité scientifique, par le biais d'interactions transversales entre laboratoires indépendants, était plus porteuse de progrès que des regroupements physiques effectifs, tendant à une homogénéisation des démarches et donc une perte de diversité et d'innovation.

8 C'est ainsi qu'en 1982, j'ai créé ma première fédération synergique (laboratoire virtuel) d'une dizaine d'équipes, appartenant au CNRS, à l'université, au MNHN et à l'Inra, dans le domaine de l'écophysiologie. Ce type d'expérience, qui m'a permis d'interagir avec des équipes très différentes, a été renouvelé en 1990 avec la création d'un club Oncogènes réunissant plusieurs équipes du campus de Jouy-en-Josas, soutenu par une AIP Inra intersectorielle.

Il restait toutefois le problème de la valorisation des résultats obtenus, et c'est ainsi que j'ai rejoint l'association Ecrin en 1993, après un long entretien avec Gérard Nominé, industriel et président du club Biotechnologies. Normalement, ce poste était destiné à un temps plein, ce que je ne voulais pas. Ecrin a accepté, sur la demande de Gérard Nominé, que je prenne le poste, en tant que bénévole, pour $20 \%$ de mon temps, sans mise à disposition par le CNRS. Je n'étais donc pas missionnée par le CNRS.

Dans ce contexte, l'enjeu était donc loin d'être fondamental pour ma carrière, d'autant plus qu'à cette époque les relations avec l'industrie n'étaient pas très bien considérées pour un biologiste. Dans les rapports d'activité que je faisais, comme tout chercheur, au CNRS, je mettais toujours, en annexe, les résultats obtenus à Ecrin, mais ceux-ci n'ont été jamais considérés dans mon évaluation scientifique.

11 La revue... Vous organisiez des groupes de travail mixtes regroupant industriels et chercheurs sur des problématiques liées au développement des biotechnologies. Qui choisissait les thèmes abordés ? La direction générale du CNRS ? La direction des sciences de la vie?

12 F.X. Je pense qu'il est nécessaire avant de répondre à ces questions, de rappeler quels sont la structure et le fonctionnement d'Ecrin. Très structurée, Ecrin a une équipe de direction solide avec un président ${ }^{1}$, un délégué général ${ }^{2}$, en permanence au siège de l'association, un conseil d'administration et son bureau jouant un rôle d'orientation stratégique et examinant les activités de l'association tous les six mois.

13 Les grandes structures d'activités d'Ecrin sont de deux sortes :

- les clubs, structures pérennes, avec au moins un président, un responsable scientifique, un chargé de mission et un bureau ;

- les actions, structures souples, de durée déterminée, pour répondre à des demandes, clairement exprimées, ponctuelles, individuelles ou collectives, d'adhérents ou de partenaires.

- Les clubs sont les véritables piliers de l'association. Le club Biotechnologies, comme d'autres clubs Ecrin, fonctionne par l'intermédiaire :

- d'enquêtes auprès de nos partenaires et adhérents ; 
- de réunions thématiques, réunissant laboratoires académiques et entreprises, ouvertes à tous, et permettant d'explorer de nouveaux thèmes et de donner accès à des sources d'informations et de compétences ;

- enfin et surtout, de groupes de travail à comité restreint, regroupant industriels et chercheurs, et où les règles de confidentialité sont respectées. communiquer, fédérer par la mise en place de consortium regroupant la majorité des acteurs français dans le domaine, labelliser en obtenant le label de qualité scientifique CNRS et valoriser par des contrats.

15 Je vais prendre un exemple précis, celui du groupe de travail (GT) «Évolution moléculaire dirigée et ingénierie des protéines ». Ce GT a été créé en 2001 suite à une réunion plénière, la première faite en France sur ce thème. Sa première action a été la rédaction d'un dossier de quatre articles dans la revue Biofutur, impliquant tous les partenaires académiques et industriels et leur permettant ainsi de mieux se connaitre, d'apprendre à travailler ensemble, et de faciliter ainsi leur fédération autour d'un projet scientifique. Ce projet a favorisé de nouvelles collaborations entre chercheurs et industriels et a permis d'obtenir des contrats. La qualité scientifique de ce GT a été reconnue par le CNRS par la création d'un groupement de recherche (GDR) en 2004. Un point à noter : ces résultats ont été obtenus très rapidement (en trois ans). et si c'était la direction du CNRS. Les thèmes étaient choisis par le bureau du club au cours de ses réunions qui de tenaient deux fois par mois. La direction du CNRS intervenait, comme les autres institutions, organismes et entreprises, par l'intermédiaire de ses représentants des départements des sciences de la vie et de chimie.

17 Je voudrais toutefois ajouter que ponctuellement des actions ont été menées directement sur la demande du CNRS. Par exemple, en 2000-2001, une action, dans le domaine biologie et technologies du vivant sur le thème "De la génomique à l'innovation industrielle », a été menée par le club Biotechnologies sur la demande de la délégation aux entreprises du CNRS. Une enquête a été menée par Ecrin auprès des entreprises de biotechnologies et une réunion plénière a été organisée entre des experts du CNRS de cette thématique et les représentants de plus d'une quarantaine d'entreprises.

tats obtenus ont permis de dégager les thèmes scientifiques à conforter ou à explorer, susceptibles d'engendrer des développements technologiques innovants et d'identifier les compétences recherchées par les entreprises industrielles. De nouveaux programmes ont été mis en place par le CNRS concernant, notamment, le criblage à haut débit et la mise en place d'une chimiothèque/ciblothèque.

19 La revue... Quel bilan dressez-vous de la dizaine d'années que vous avez passées à Ecrin en tant que responsable scientifique des biotechnologies?

F.X. Un bilan très positif, pour une activité que je menais en tant que bénévole à $20 \%$ de mon temps, pour les raisons suivantes:

- l'organisation de plus d'une douzaine de réunions thématiques et forums sur les grands thèmes d'actualité de ce domaine, réunissant de 5 à plus de 200 personnes, comme ce fut le cas pour le forum sur la propriété industrielle en biotechnologie, organisé en partenariat avec le ministère de la Recherche ;

La revue pour l'histoire du CNRS, 17 | 2007 
- la création et la coordination d'une quinzaine de groupes de travail actifs et pragmatiques, réunissant en moyenne une dizaine de laboratoires appartenant aux grands organismes de recherche et 5 à 6 entreprises industrielles, afin d'élaborer des programmes de recherche pour des collaborations recherche publique et entreprises et d'obtenir des contrats ;

- la mise en place et l'animation scientifique de réseaux d'experts, véritables consortiums impliquant des équipes des différents organismes de recherche publique et des entreprises, autour de projets scientifiques sur des thèmes insuffisamment abordés ou approfondis en France, pour le développement de méthodologies innovantes. De tels consortiums permettent d'avoir une meilleure visibilité de ce qui se fait en France et par qui, une des premières requêtes faites par les entreprises. Deux de ces réseaux sont devenus des GDR du CNRS. Je vous ai déjà cité le réseau sur l'évolution moléculaire des protéines, le deuxième porte sur l'immunociblage des tumeurs ;

- la valorisation des résultats par des contrats public et public/privé, plus de six ces dernières années, en réponse à des appels d'offres du CNRS et du ministère de la Recherche.

La revue... Pensez-vous que le CNRS n'a pas assez ou pas su utiliser les compétences du club Ecrin Biotechnologies?

F.X. Il est vrai que l'on pense toujours que cela aurait pu être mieux, surtout après avoir fait un bilan portant sur plus de dix ans. Les raisons sont presque toujours les mêmes : manque de visibilité et de dialogue. Nous faisions régulièrement des rapports d'activité, mais les retours d'évaluation, au niveau des clubs, et quand il y en avait, étaient trop généraux et pas assez constructifs.

La revue... Que conseilleriez-vous aux jeunes arrivant sur le marché du travail ?

F.X. C'est une question très intéressante à laquelle j'ai dû répondre lorsque j'ai présidé une table ronde sur les micro et nanotechnologies au salon des formations et métiers scientifiques à la Cité des sciences et de l'industrie en janvier 2005. Cette table ronde était destinée à des ingénieurs, des étudiants en fin d'étude universitaire, des doctorants et des post-doctorants.

Le public était très nombreux et les échanges très fructueux, ce qui montre bien que les jeunes scientifiques sont de plus en plus préoccupés par leur intégration dans le monde du travail. Les jeunes hésitent à faire une thèse et encore plus un post-doctorat. On forme des jeunes, on finance leur instruction. On leur demande de passer trois ans aux États-Unis et quand ils reviennent, ils n'ont pas de poste, ils ne peuvent et ne veulent plus faire de recherche.

Cette table ronde a débuté par une présentation de ces technologies et de leurs impacts dans le domaine de la biologie et de la médecine. Le débat s'est ensuite ouvert sur les nouveaux métiers qu'offraient ces nouvelles technologies et ce qu'ils nécessitaient comme formation. En fait, le point le plus important qui s'est dégagé de toutes les discussions, c'est que, désormais, on ne peut plus se permettre de n'avoir qu'une seule spécialité dans son bagage scientifique et que l'on doit impérativement se tourner vers une pluridisciplinarité de sa culture scientifique et technologique.

La revue... Quel bilan tirez-vous de votre expérience à Ecrin et quels sont vos projets?

F.X. Un bilan finalement très positif. J'ai été très heureuse de mettre en place et de diriger au sein d'Ecrin toutes ces activités de Biologie, biotechnologies et de santé. J'ai beaucoup donné, mais j'ai aussi beaucoup reçu et beaucoup appris. Le développement de plus en plus important des activités de biotechnologies à Ecrin, qui s'est traduit par 
la transformation du club en un domaine et la création d'un pôle de Biologie intégrative et une surcharge énorme de travail, m'a fait prendre la décision de me retirer d'Ecrin à la fin de l'année 2005.

Je consacrerai alors tout mon temps à mes activités de recherche, que je mène depuis le début de l'année 2005 au Laboratoire de mathématiques appliquées aux systèmes à l'École centrale Paris, par mise à disposition par le CNRS, pour participer au développement de l'interface biologie/modélisation mathématique et des relations industrielles.

D'après des propos recueillis le 28 avril 2005 par Denis Guthleben, Daniel Pardo et Girolamo Ramunni. Et des compléments d'information apportés par Françoise Xavier.

\section{NOTES}

1. 1. Depuis la création d'Ecrin, les présidents ont été : Michel Pecqueur (janv. 1991-nov. 1995), Jean-Jacques Duby (nov. 1995-déc. 1998), Bernard Pache (déc. 1998-déc. 2005), Guy Paillotin (déc. 2001-déc. 2005), Jean-Jacques Doyen (depuis déc. 2005).

2. 2. Trois délégués généraux se sont succédé depuis la création d'Ecrin : Serge Gross (janv. 1991-fév. 1996), Jacques Martineau (fév. 1991-nov. 2003), Christian Ngô (depuis novembre 2003).

\section{RÉSUMÉS}

Françoise Xavier a été pendant treize ans responsable scientifique du club Biotechnologies puis du domaine «Biologie, biotechnologies et santé » à l'association Ecrin (Échange et coordination recherche-industrie). Elle nous fait part de son expérience en revenant sur une thématique qu'elle connaît bien : les biotechnologies.

For thirteen years, Francoise Xavier had been in charge of the club "Biotechnologies" and then responsible for the "Biology, biotechnologies and health" field within ECRIN association (Exchange and Collaboration Research-Industry). She makes us share some of her experience of a thematic she knows well: biotechnologies. .

\section{AUTEUR}

\section{FRANÇOISE XAVIER}

Françoise Xavier, docteur ès sciences, est directrice de recherche au CNRS. Elle se consacre actuellement au développement de l'interface biologie/modélisation 
mathématique et des relations industrielles au Laboratoire de mathématiques appliquées aux systèmes à l'École centrale Paris. 